\title{
Weighted Multiple Linear Regression Model for Mobile Location Estimation in GSM Network
}

Longinus Sunday Ezema, Electrical and Electronic Department, University of Technology, Owerri, Imo State, Nigeria Cosmas I. Ani, Electronic Department, University of Nigeria Nsukka, Enugu State, Nigeria

\begin{abstract}
The numbers of crimes and accidents, among other challenging issues, requiring a mobile application with localization capabilities are on the increase. Yet there is under-utilization of location information provided by mobile phones. The accuracy and cost of implementation of mobile position localization on cellular network have been an issue of research interest. In this paper, the statistical modelling of mobile station (MS) position location was carried out using weighted multiple linear regressions (WMLR) method. The proposed statistical modelling approach was based on received signal strength (RSS) technique. The model improved localization accuracy. The model's simulated results were analysed and compared with the existing MLR using real measured data collected from GSM network in a light urban environment in Enugu, Nigeria.
\end{abstract}

\section{KEYWORDS}

Cellular Network, FCC USA, Mobile Station, Mobile Telecommunication Network, Nigeria, Radio Location, Received Signal Strength, Statistical Model

\section{INTRODUCTION}

Mobile location estimation means defining the coordinates of a mobile station (MS) in two or three dimensions - latitude, longitude and altitude. Localization of mobile station has been attracting interests not only from the telecommunication system vendors, network operators and mobile network users. This is owing to its potential base and other associated location-based services. Such location base services include logistic, fleet management, entertainment, tourism, safety and security, and among others (Ezema \& Ani, 2016). Therefore, mobile phones are not just used only for making calls and sending messages.

Localization can be carried out by the application of some measured signal properties as signal propagates from mobile station to base station (BS) or vice versa. Such notable signal properties include received signal strength (RSS), uplink-time of arrival (U-TOA), enhanced observed time difference (EOTD)/ observed time difference of arrival (OTDOA), time advance (TA)/ round trip time (RTT) and angle of arrival (AOA)/direction of angle (DOA) (Surendra, Reenu and Chun, 2019).

\section{DOI: 10.4018/IJITN.2020010105}

This article published as an Open Access article distributed under the terms of the Creative Commons Attribution License (http://creativecommons.org/licenses/by/4.0/) which permits unrestricted use, distribution, and production in any medium, provided the author of the original work and original publication source are properly credited. 
Recently, the combinations of measured properties are used to enhance the accuracy of localization. Cell Identification (CID) as a parameter is mainly used in hybrid technique though it has relative low accuracy when used alone. These parameters are called location dependent parameters (LDP) (Ezema \& Ani, 2017, Shang, et al., 2015, Venkatraman and Caffery, 2004). There are three different localization methods - geometric, artificial intelligent and statistical methods. The methods have been applied directly on the LDP for MS positioning with varying degree of accuracy.

There are two basic types of networks that originate the location parameters - the space segment (satellite in space) and the earth segment networks. The satellite in space usually referred to as global positioning system (GPS) is a conventional technique used for locating position over the years (Brena. et al. 2017). The improvements on the performance of the GPS brought about by the evolution of both A-GPS and D-GPS heavily relied on the cellular network infrastructure (Mukherjee \& Biswas, 2018).

Most of the cellular traditional methods for localization have been developed to operate under line-of-sight (LOS) propagation mode. NLOS error mitigations have been the major challenge of localization in cellular network (Qi, Kobayashi, and Suda, 2006). Most of the performance criteria have been standardised by Federal Communication Commission (FCC) of United State of America (USA), a communication regulatory body in the US. The phase III of FCC accuracy requirements and regulations released in February 2015 are to be enforced on the USA mobile operators by 2021.The accuracy requirement of phase III is yet to be reached. The application such as emergency service (E-911 \& E-112) with high demand for accuracy, unlike commercial applications, is the driving force for phase III accuracy requirement (Ezema \& Ani, 2017; Zeytinci, Sari, Harmanci, Anarim and Akar, 2013; Tong, 2015).

\section{REVIEW OF RELATED WORKS}

In the field of mobile application, location base services have been an interesting topic for researchers. The following research works in mobile location in GSM network have been reported in journal publication and reviewed in this section.

Mobile location estimation using an interpolative neural network was proposed in (Chien-Sheng, Jium-Ming, and Chin-Tan, 2013). It used three angles of arrival measurement to achieve a better result than the analytical (the weighted average and optimal position) in the presence of noisy and NLOS measurement error. The neural network uses its ability to memorize and generalize data to interpolate the measured angles to estimate the mobile position. In order to avoid over-fitting of the network, the training of the neural network was done with ideal patterns gotten from the mathematical relationship that exists between the angles of arrival and the mobile position. The performance of this technique is highly dependent on the noisy and NLOS measurements of AOA, and the mobile propagation environment.

In Caffery and Stuber (1998), the RSSI-based technique because of its coverage, availability, easy of measurement and requiring no additional hardware installation have become the ideal method for GSM and other cellular localization. Here, the author proposed a data fusion algorithm of Pearson's correlation coefficient (PCC) with RSSI and other basic BS information to estimate the position of a mobile station. The performance of the technique when implemented with an ordinary mobile phone showed a maximum error of $550 \mathrm{~m}$ and mean error below $150 \mathrm{~m}$. This technique showed good robustness against change in environment. It was easy to be implemented with less mathematical complexity and required no previous statistical knowledge of the MS. The accuracy of this technique is below the accuracy requirement of FCC of United State of America and shouldn't be used for emergency services like E-911 and E-112.

A localization algorithm based on RSSI and basic BS information like the coordinate of the BS to determine the MS position was proposed by Takenga and Kyamakya (2007). In this research, the coordinates of the intersection of the line of bearing (LOB) was averaged out. Having more than two variable estimations based on other estimated values hampered the accuracy of the technique. This 
was probably done to avoid the cost of installing highly directional antennas which could have helped improve the accuracy of the techniques. Though the addition of hardware makes it less attractive for deployment in large area it will improve the accuracy. It would have been better to adopt a selective algorithm to pick only the point of intersection closer to the true MS position than the whole algorithm adopted by the authors. The selective algorithm could have eliminated the BSs seriously affected by NLOS propagation.

CellSense, a probabilistic algorithm based on RSSI-fingerprint for MS localization was proposed by Shaukat, Ansari, Farooq, Ibrahim and Faisal (2009). By applying gridding approach, the authors were able to reduce the overhead of constructing fingerprint database at the expense of reduced resolution. The CellSense algorithm used Baye's theorem to calculate the probability of MS being in each of the fingerprint location and return as the MS position the location with maximum probability. In order to increase the accuracy, the system determined the MS position by weighted average of the $\mathrm{K}$ most probable fingerprint location. The CellSense-Hybrid techniques also proposed to maintain the accuracy and reduce the computational complexity of the CellSense. To achieve this, the cell grid size was lowered and a deterministic refinement phase added. The accuracy of these two techniques showed that the probability of locating a mobile device with $70 \mathrm{~m}$ is $67 \%$ and $273 \mathrm{~m}$ is $95 \%$ for CellSense while for CellSense-Hybrid is $75 \mathrm{~m}$ and $290 \mathrm{~m}$ for $67 \%$ and $95 \%$, respectively.

In this paper, MLR analysis was adopted in modelling the relationship between independent variable -RSS and dependent variable -MS coordinates by fitting a linear equation to observed data. The method of least-squares was adopted for fitting a regression line. Minimizing the summed squared of residuals of actual and estimated location is the reason behind the success of regression techniques. In the order to enhance the accuracy, the RSS from the serving cell sectors were weighted to have more determining power than RSS values from neighbouring sectors.

\section{IMLR MODELLING APPROACH}

This section presents the approaches adopted in the development of the IMLR model for mobile positioning in GSM network. These include all the processes ranging from data collection to model development. The approaches explained here are based on two phases - the offline phase or the sites survey and data collection phase, and the on-line phase where the real-time estimation of MS location was carried out.

\section{Field Measurement}

In this research, empirical and real-time analysis approaches were adopted. The site survey conducted showed that the area of study which is $5 \mathrm{~km} \times 4 \mathrm{~km}$ is a light urban based on characteristics of the environment. These characteristics include the population, the BS cluster, the building height and among other factors. The drive test routes were also chosen at the time of site survey to ensure no route was taken twice. Several materials were utilised in measuring and recording data from base stations within a cell cluster. The method used depends greatly on the network coverage size and the parameters adopted. The drive test employed for data collection and data analysis was carried out in idle mode. A van carrying the drive test set-up in Figure 2 traveled a predetermined route in Figure 1 at a fairly fixed speed of $30 \mathrm{Km} / \mathrm{h}$ with $\mathrm{MS}$ antenna height of $1 \mathrm{~m}$ above the ground. The GPS receiver antenna is mounted on the roof of the van due to the line-of-sight limitation of GPS system. The TEMS software in Figure 3 at every measurement point records the MS RSS and the GPS coordinates. The mobile network chosen for the research is Mobile Telecommunication Network (MTN) Nigeria.

During data analysis, any measurement with GPS indicating visibility to two or fewer satellite transmitters was filtered out from the databases. This is because GPS makes use of TOA from satellites to form a trilateration with the intersection of these circles the actual mobile position. For this reason, and for accurate 2D localization, GPS should be visible to at least three satellites transmitters in space. Most mobile receivers have a minimum sensitivity value of $-103 \mathrm{dBm}$ (Fara and Phil. 2015). 
Figure 1. Show the working area of Achara Layout including Achara Layout Primary School

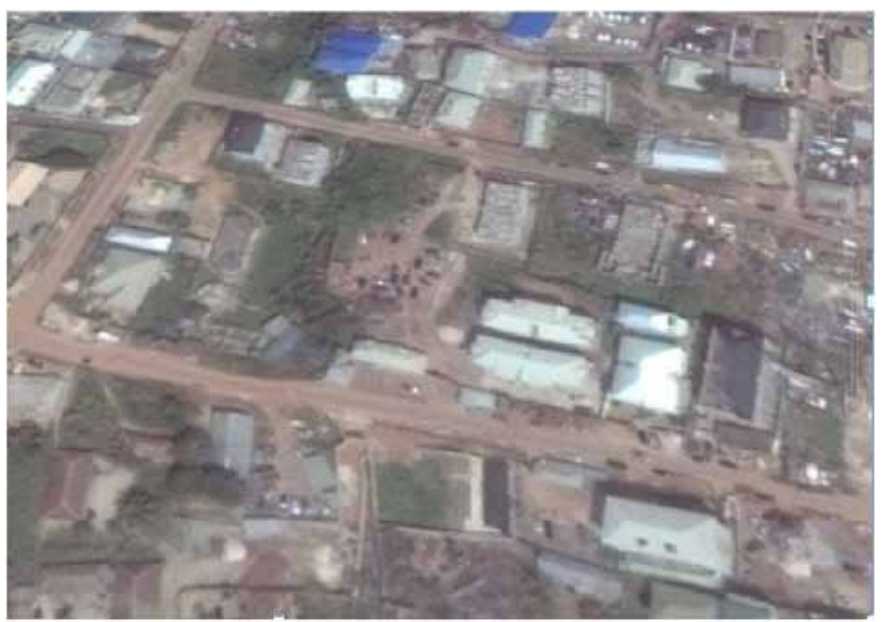

Figure 2. Show the drive test connection picture

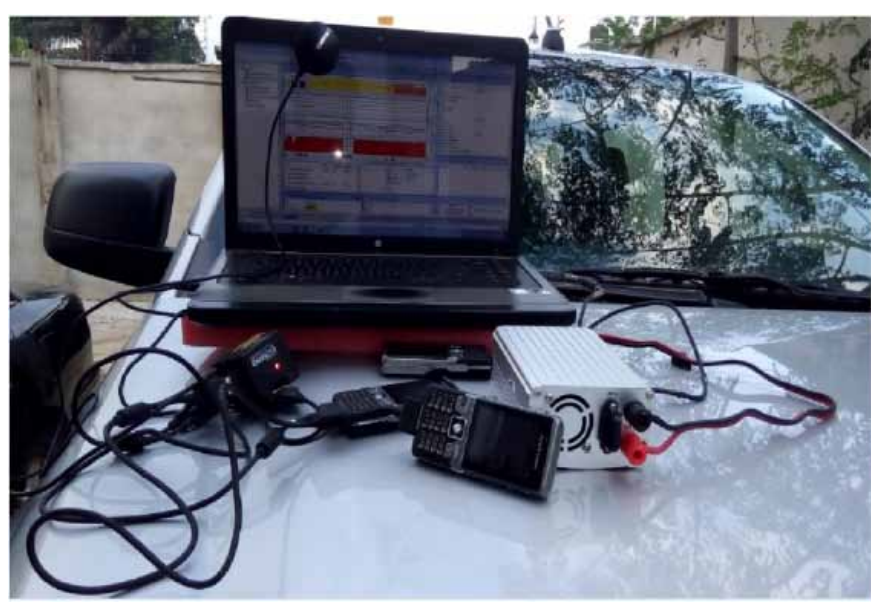

This value was assumed in cases where there are no signal strength measurements for a base station or a cell sector while signals below that were truncated.

\section{Multi Linear Regression Analysis}

A multiple linear regression model is a linear model that explains how one variable (y-variable) relates to two or more variables. The relationship between the response (dependent) variable (Y) and the explanatory (independent) variables $(\mathrm{X} 1, \mathrm{X} 2, \ldots, \mathrm{Xn})$ is established by fitting a linear equation to observed data if it is established that there is a relationship between the variables in equation. It does not necessarily mean that one variable causes the other. The most widely used method of fitting a regression line is by the method of least-squares. However, regression techniques try to minimise the summed squared of residuals of the real and the estimated by method of least-squared algorithm. The regression coefficients that achieve this result is obtained by the least-squared algorithm. The general equation of multiple linear regression model can be expressed as (Chu, $\mathrm{Ng}$, \& Leung, 2006; Ezema \& An, 2016): 
Figure 3. Demonstrate the TEMS software showing the working area, the base station, and network parameters

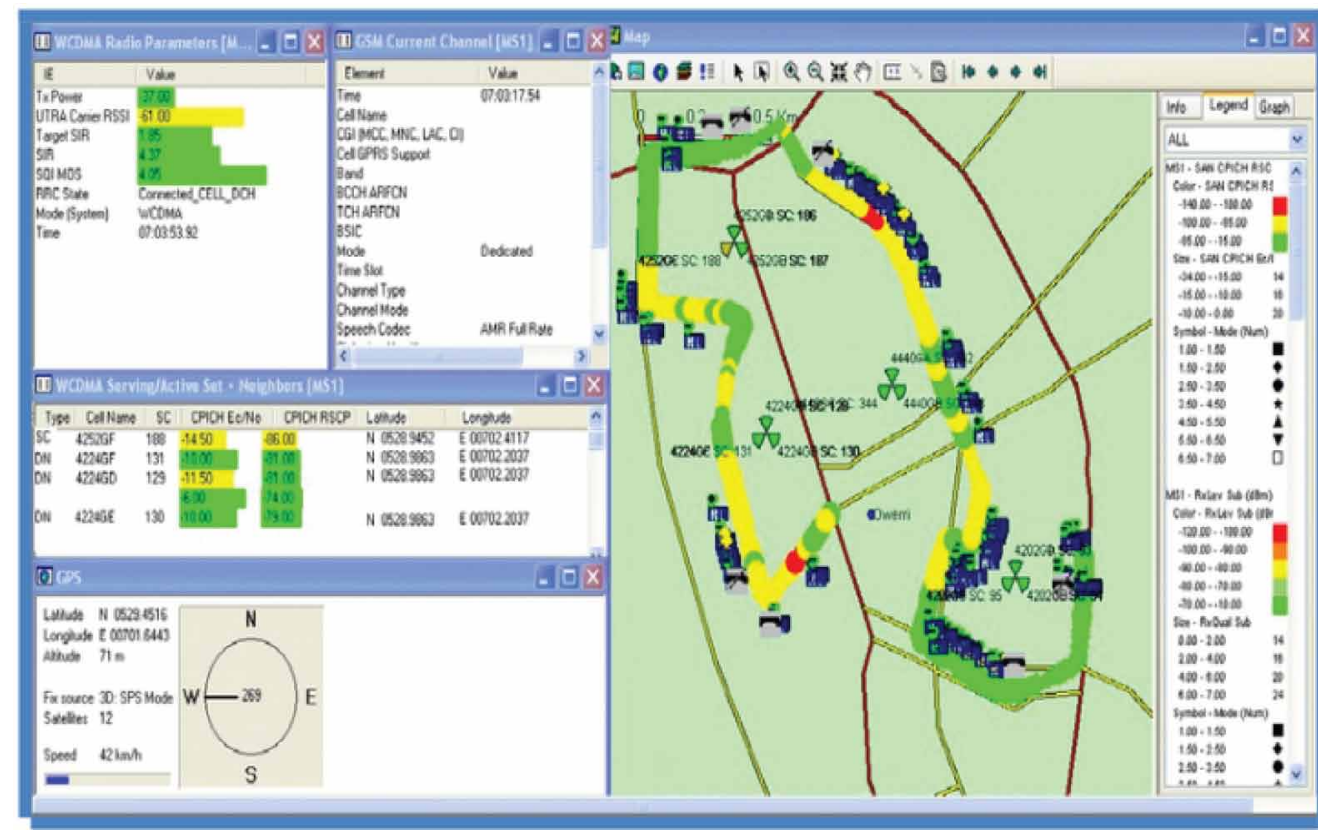

$y=A+B_{1} x_{1}+B_{2} x_{2}+\ldots+B_{n} x_{n}+\epsilon$

where $\mathrm{A}$ is the intercept, $\mathrm{B} 1, \ldots, \mathrm{Bn}$ are slopes and unknown constants associated with $\mathrm{x} 1, \ldots, \mathrm{xn}$, $\epsilon$ is a random error component or disturbance. The error term $\epsilon$ contains factors unaccounted for that affect Y. No matter the number of independent variables included in a model, there will always be factors we cannot include, these factors are jointly contained in $\epsilon$. It is normally assumed that the errors are uncorrelated and have mean zero and an unknown $\sigma 2$ (Pandis, 2016; Iserbyt, Schouppe and Charlier, 2015).

For a model with three explanatory variables, the relationship between $\epsilon$ and the explanatory variables (x1, x2, x3,), is given in Equation 2 (Zhou. and Kee-Yin Ng. 2006):

$E\left(\epsilon \mid x_{1}, x_{2}, x_{3}\right)=0$

Now, relating this to the mobile location estimation where there are two unique variables (received signal strength and location coordinate). Let the location of the MS be $\left(x_{M}, y_{M}\right)$ and this location information measured $\left(x_{i}, y_{i}\right)_{i=1}^{m}$ as well as the received signal strengths (R) of many mobile stations within the target area be stored in a database. The regression equation can be formulated as:

$\left\{x_{i}\right\}_{i=1}^{m}=b_{0}+\sum_{j=1}^{N} b_{j} R_{i j}+\varepsilon$ 


$$
\left\{y_{i}\right\}_{i=1}^{m}=c_{0}+\sum_{j=1}^{N} c_{j} R_{i j}+\varepsilon
$$

where, $\mathrm{b} 0$ and $\mathrm{c} 0$ are constants and $\mathrm{bj}$ and $\mathrm{cj}$ are the vectors (multiple) regression coefficients of $\mathrm{x}$ - y coordinates, Rij is the RSSI of jth BS at location $\mathrm{i}, \varepsilon$ is the error term, and $m$ is the number of locations (training data length). It is much easier to perform least-squares analysis using matrix algebra. Therefore, Equations 4 and 5 can be written in matrix form without error term as given in Equations 4 and 5:

$$
\underline{X}=\underline{Q B}
$$

is equivalent to:

$$
\begin{aligned}
& {\left[\begin{array}{l}
x_{1} \\
x_{2} \\
\vdots \\
x_{m}
\end{array}\right]=\left[\begin{array}{lllll}
1 & R_{11} & R_{12} & \ldots & R_{1 N} \\
1 & R_{21} & R_{22} & \ldots & R_{2 N} \\
& & \vdots & & \vdots \\
1 & R_{m 1} & R_{m 2} & \ldots & R_{m N}
\end{array}\right] \times\left[\begin{array}{l}
b_{0} \\
b_{1} \\
\vdots \\
b_{N}
\end{array}\right]} \\
& \underline{Y}=\underline{Q \hat{C}}
\end{aligned}
$$

is equivalent to:

$$
\left[\begin{array}{l}
y_{1} \\
y_{2} \\
\vdots \\
y_{m}
\end{array}\right]=\left[\begin{array}{lllll}
1 & R_{11} & R_{12} & \ldots & R_{1 N} \\
1 & R_{21} & R_{22} & \ldots & R_{2 N} \\
& & \vdots & & \vdots \\
1 & R_{m 1} & R_{m 2} & \ldots & R_{m N}
\end{array}\right] \times\left[\begin{array}{l}
c_{0} \\
c_{1} \\
\vdots \\
c_{N}
\end{array}\right]
$$

The number of the measurement points (observations $-(\mathrm{m})$ ) must exceed the number of RSS (independent variables $-(n)$ ) by at least two (i.e. $m>n+2$ ) to a good prediction without over generalisation. The least-square method tried to minimize $\sum e_{i}^{2}$ in order to find the coefficient ( $\hat{B}$ and $\hat{C}$ ) of the regression equation as shown in Equations $6 \mathrm{a}$ and $6 \mathrm{~b}$ (Zhou, and $\mathrm{Ng}, 2006$; ChienSheng, 2017; Walpole and Myers, 1985):

$$
\hat{B}=\left(\underline{Q^{T}} \underline{Q}\right)-1 Q^{T} X
$$

similar to that:

$$
\hat{C}=\left(\underline{Q^{T}} \underline{Q}\right)-1 Q^{T} Y
$$


Having determined the coefficients of the regression equation in Equations 6 ( $a$ and $b$ ), the location of the target MS can be obtained using Equation 7:

$$
\left.\begin{array}{c}
X_{M}=[1 \underline{R}] \cdot \hat{B} \\
Y_{M}=[1 \underline{R}] \cdot \hat{C}
\end{array}\right\}
$$

\section{Development of Weighted MLR (WMLR)}

To improve the multiple regression analysis, the highest measured value of RSS for every measurement point is assumed to come from the serving base station. These values were weighted to have more determining power than values from neighbouring base station sectors. The weighted MLR technique was achieved by creating a weighted matrix A from the matrix Q in Equations 4 and 5. This was formed with the measured received signal strength by replacing the highest value of each row with one (1) and the rest with zero (0). The highest value in each row was assumed to emanate from the serving base station sector where the mobile station to be located resides.

The independent variable for the WMLR is formulated as given in Equation 8:

$\mathrm{L}=\mathrm{Q}+\mathrm{A}^{*} \mathrm{~W}$

where $\mathrm{w}$ is a scalar value called multiplier.

Matrix L was substituted for matrix Q in Equations 3-6 to arrive at the Weighted MLR models shown in Equations 11a and 11b. A multiplier (w) was chosen at random to change the value of the weighted matrix until an optimum value was attained. The optimum value of the multiplier was found to be 10 .

Through the application of Matlab codes, the mathematical processes expressed as in Equations $3-6$, the coefficients of regression equations were gotten using Equation 7 and substituted in Equation 3 to form Equations 9a and 9b. These Equations (9a and 9b) developed are the model equations for mobile location estimation in the area under consideration:

$$
\begin{aligned}
& \text { Latitude }=y=\left(\begin{array}{l}
625330+3.62 R_{1}+0.24 R_{2}+0.41 R_{3}-0.87 R_{4}+0.53 R_{5}+1.22 R_{6} \\
-1.01 R_{7}-2.78 R_{8}+2.78 R_{9}-1.73 R_{10}+0.52 R_{11}-0.04 R_{12}
\end{array}\right) \\
& \text { Longitude }=x=\left(\begin{array}{l}
729253-0.067 R_{1}-1.98 R_{2}+3.2 R_{3}+0.74 R_{4}-0.62 R_{5}-2.64 R_{6} \\
+0.61 R_{7}+0.14 R_{8}-0.51 R_{9}-5.56 R_{10}-1.64 R_{11}+0.32 R_{12}
\end{array}\right)
\end{aligned}
$$

Equations 9 ( $\mathrm{a}$ and $\mathrm{b}$ ) are the proposed weighted multiple linear regression models. The numerical constants in Equations 9 ( $\mathrm{a}$ and $\mathrm{b}$ ) are gotten from Equations 6 ( $\mathrm{a}$ and $\mathrm{b}$ ) by substituting $\mathrm{L}$ for $\mathrm{Q}$. The constants represent the $\hat{B}$ and $\hat{C}$ which stand for partial coefficients of the regression equations. The first numerical constants are the intercept while the rest are the slopes of the regression line that minimized the root mean square error.

\section{SIMULATION AND RESULT ANALYSIS}

In this section, the WMLR model was simulated using real data collected from field measurement from some of GSM BS that cover the urban area of Achara layout in Enugu is presented. These evaluations include performance evaluation of the weighted multi linear regression model; the impact 
of number of base stations on the proposed technique accuracy and proposed technique with US FCC accuracy benchmark. The performance matrix considered for the evaluation include the root mean square error (RMSE), the cumulative distribution frequency (CDF) and percentage of positioning error (67\% and 95\%) as recommended by United State Federal Communication Commission (FCC, 2014; Leong et al., 2017; NovAtel, 2003; Reed et al., 1998).

\section{WMLR Estimation Simulation and Results}

The simulation result was carried out using three hundred and ninety-seven (397) measurement points and twelve cell sectors from four (4) base stations detected by the MS. Two hundred and seventy-nine measurement data (279) were used for model formulation while $30 \%$ of the data were applied to the model for performance evaluation. The GPS coordinates at each measurement points were used for error calculation and validation. The MATLAB software was used to determine the regression coefficient through the mathematical processes of model formulation and simulation. Figure 4 showed the CDF of the MS estimated errors for the WMLR. Figure 5 showed how closely the estimated path followed the real path.

The simulation results of the weighted MLR locator showed that the root mean square errors, $67 \%$ of estimated location error (ELE) and 95\% of ELE do not exceed $53 \mathrm{~m}, 62 \mathrm{~m}$ and $106 \mathrm{~m}$ respectively. The performance of WMLR was enhanced when the received signal from the serving sectors were weighted to have more determining power than the RSS from the neighbouring sectors. The descriptive statistic presented in Table I also agreed to the fact that WMLR showed high localisation accuracy. The study area was considered as a light urban and that makes the result even more interesting considering the accuracy achieved.

Figure 5 is the curves of the real and estimated path of drive test route. The longitude was plotted against the latitude for the GPS measured coordinate and the estimated coordinate. The plot showed how closely the estimated path followed the real path which indicates how good the performance of the proposed model is.

Figure 4. CDF of the MS estimated error using the proposed model

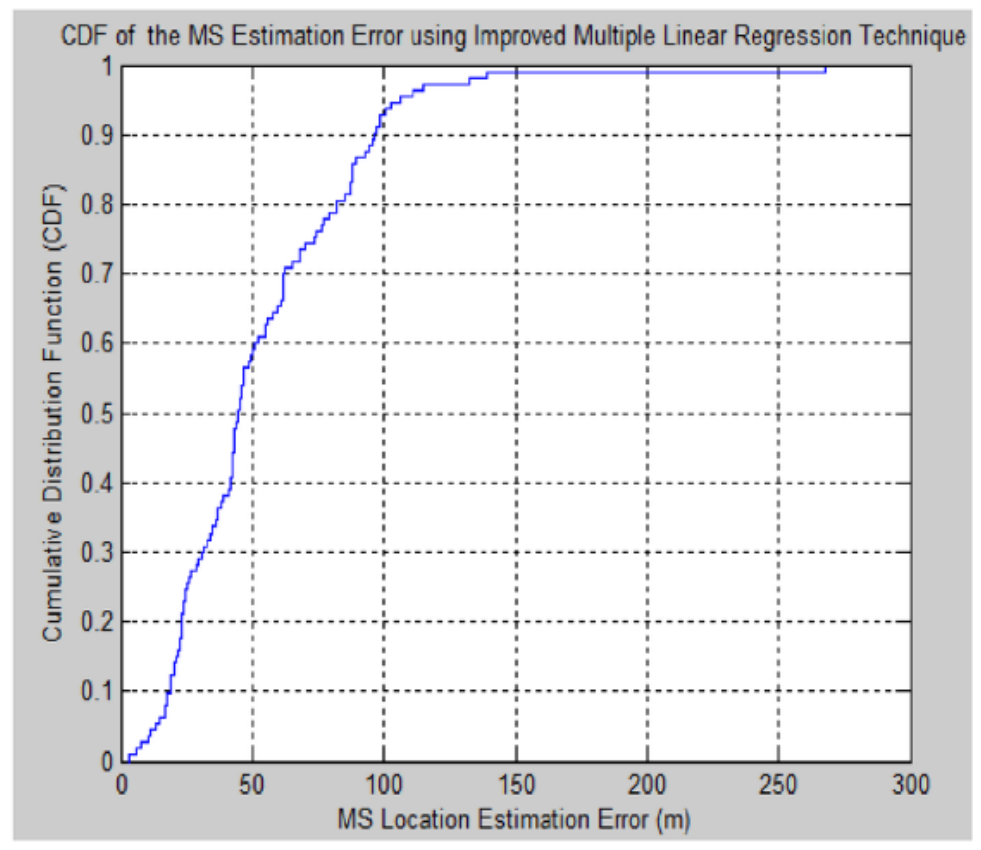


Figure 5. Real (red) and estimated path (blue) of a drive test through the centre of Achara layout

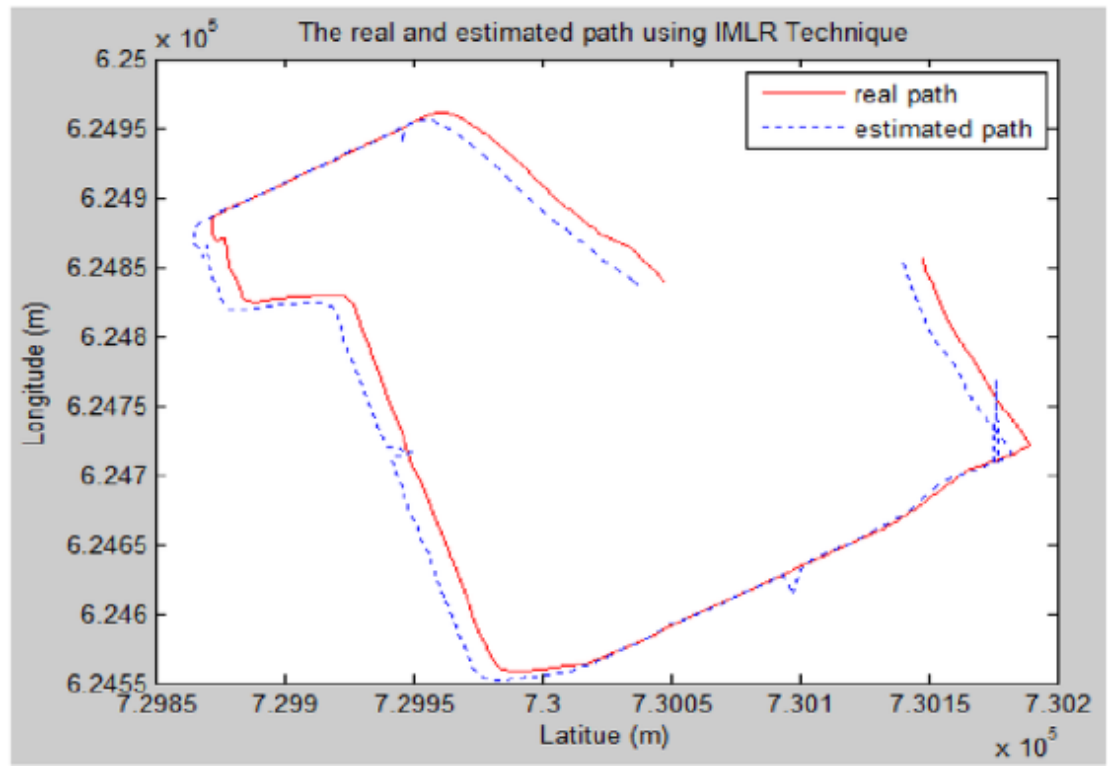

\section{Performance Comparison of WMLR With MLR and US FCC Accuracy Benchmark}

The statistical parameters in Table 1 proved that weighted MLR outperformed MLR and both meet the FCC accuracy benchmark requirement. WMLR and MLR showed a consistent result for each run and take few seconds to converge. The table compared the simulation result of the proposed WMLR model over MLR, and the FCC maximum error standard for network-based techniques.

It can be seen in Figures 4 and clearly in Table 1 that the weighted MLR performed excellently showing 38\% and 65\% improvement over the FCC benchmark for $67 \%$ and $95 \%$ error estimation respectively. This is the accuracy requirement for mobile locator to be considered good for emergency services.

\section{Effect of Number of BS on Result}

The numbers of hearable base stations formed an overlapped area dimension. Throughout the simulation, the only varying variable was the number of cell sectors. Using the same model technique, the performance of 4,8 and 12 cell sectors were evaluated. It is evidence from graph that the distributions of the curves are quite similar. For every four (4) cell sectors added, the accuracy was improved by almost $25 \%$. The 8 cell sectors errors practically shifted $6 \mathrm{~m}$ on average compared to 12 cell sectors. The 4 cell sectors on average shifted $45 \mathrm{~m}$ and $34 \mathrm{~m}$ compared to 12 and 8 cell sectors respectively. This implied that a further reduction in cell sector will give a very poor result. However, Figure 6 showed that the localization

Table 1. Performance analysis of WMLR with FCC maximum error standard

\begin{tabular}{|l|l|l|l|}
\hline & FCC & IMLR & \% Improvement \\
\hline $67 \%$ & 100 & 62 & $\mathbf{3 8}$ \\
\hline $95 \%$ & 300 & 106 & $\mathbf{6 5}$ \\
\hline RMSEr & - & 53 & \\
\hline
\end{tabular}




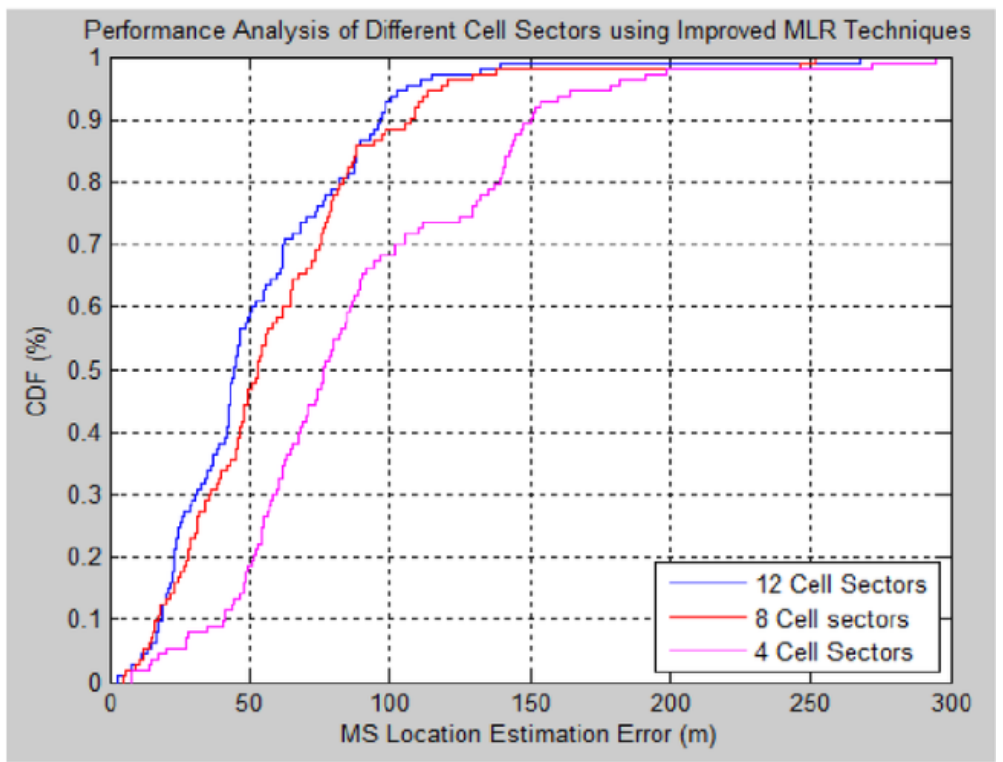

accuracy of the WMLR model deteriorated as the numbers of cell sectors are reduced. Thus the result achieved can be greatly improved on if the area under consideration was dense urban. This is because urban areas usually have BSs with less coverage area. Therefore, more cell sectors would have been detected if the same area was covered in urban area than obtained for the study. Therefore, the type of environment seriously impacts on the performance of RSS techniques.

In spite of the reduction in a number of sectors, the positioning accuracies for the reduced cell sectors still achieved a very good performance relative to the FCC standard, and therefore, can be applied for E-911 emergency services with high accuracy requirement.

\section{Other Performance Analysis}

Location accuracy criteria was used to evaluate the performance of the work in the above section which has been the major index of consideration for emergency utilization such as E-911 as established by US FCC accuracy benchmark (FCC, 2014). However, there exist other performance indices worthy of consideration as highlighted below.

This technique achieves high accuracy at a relatively low cost of implementation. It requires no additional hardware and signalling with no network and handset upgrade required as in most techniques. It hardly fails or gives false location as the location parameter (RSS) is based on standard network measurement reports and because it is readily available in all mobile communication networks. The technique is computationally less intensive. The processing time or time-to-first-fix (TTFF) is less than 10 seconds. The physical constraint and requirements related with the implementation and usage of mobile location technology such as processing load, cost, signal load, power consumption, and hardware and software size are not an issue of concern with the technique. This makes the technique more reliable, applicable and practical to be considered for standardisation and commercialisation for all location base services (LBS) especially emergency LBS. 


\section{CONCLUSION}

This paper presented a weighted Multiple Linear Regressions (WMLR) model for mobile positioning based on RSS. The multiple linear regression was greatly improved by adding weight to the received signal strength of the serving cell sectors. The mechanism was implemented to increase the determining power of the serving sectors more than the neighbouring sectors. The simulation results of the weighted MLR locator showed that the root mean square error is $53 \mathrm{~m}$, and the $67 \%$ of the estimated location error and $95 \%$ of ELE do not exceed $62 \mathrm{~m}$ and $106 \mathrm{~m}$ respectively. The achieved location accuracy surpassed the USA FCC benchmark requirement $(67 \%$ error of $100 \mathrm{~m}$ and $95 \%$ error of $300 \mathrm{~m})$ for a network-based technique. The models can be implemented for an emergency application like E-112 and E-911 as well as other location-based services. This new positioning approach that can be implemented in any cellular networks without additional network infrastructure is seen as a great added advantage. Also, easy of measurement and high availability of RSS in all cellular networks gave this method an edge over all other methods using different LDPs. 


\section{REFERENCES}

Al-otaibi, F. D. (2007). A Real Time Intelligent Wireless Mobile Station Location Estimation with Application to TETRA Network. King Saud University College of Engineering, Electrical Engineering Department.

Brena, R.F., García-Vázquez, J.P., Galván-Tejada, C.E., Muñoz-Rodriguez, D., \& Vargas-Rosales, C.; Fangmeyer. (2017). J. Evolution of Indoor Positioning Technologies: A Survey. Journal of Sensors. doi:10.1155/2017/2630413

Caffery. J and Stuber. G, (1998), Overview of radiolocation in CDMA Cellular System. Journal of IEEE Communication Magazine, 36(4).

Che, C.-S. (2017). A Non-line-of-sight Error Mitigation Method for Location Estimation. International Journal of Distributed Sensor Networks, 13(1). doi:10.1177/1550147716682739

Chien-Sheng, C., Jium-Ming, L., \& Chin-Tan, L. (2013). Neural Network for WGDOP Approximation and Mobile Location, Hindawi Publication Corporation. Mathematical Problems in Engineering.

Chu, K. M. K., Ng, J. K. Y., \& Leung, K. R. (2006, April). A new approach for locating mobile stations under the statistical directional propagation model. In Proceedings of the 20th International Conference on Advanced Information Networking and Applications (AINA'06) (Vol. 1, pp. 932-940). IEEE.

Ezema, L. S., \& Ani, C. I. (2016). Multi Linear regression Model for Mobile Location Estimation in GSM Network. Indian Journal of Science and Technology, 9(6). doi:10.17485/ijst/2016/v9i6/75195

Ezema, L. S., \& Ani, C. I. (2017). Artificial Neural Network Approach to Mobile Location Estimation in GSM Network. International Journal of Electronics and Telecommunications, 63(1), 39-44.

Federal Communication Commission (FCC). (2014), Wireless E911 Location Accuracy Requirements. Federal Register. Retrieved from https://www.federalregister.gov/documents/2014/03/28/201406618/wireless-e911location-accuracy-requirements

Iserby, P., Schouppe, G., \& Charlier, N. (2015). Multiple Linear Regression Analysis of Factors Affecting the Simulated Basic Life Support (BLS) Performance with Automated External Defibrillator (AED) in Flemish Lifeguards. Official Journal of the European Resuscitation Council, 89, 70-74. PMID:25636894

Leong, L., Matthew, J., Gregory, S. R., Sam, J. B., Arisha, C. M., Bianca, L. M., \& Michael, J. W. (2017), Comparison of Accuracy and Precision of GPS-Enabled Mobile Devices. In Proceedings of the 2016 IEEE International Conference on Computer and Information Technology (CIT). doi:10.1109/CIT.2016.94

Mukherjee, S., \& Biswas, G. P. (2018, August 26). (20-18), Location Estimation based Routing for Mobile Adhoc Network. Journal of Intelligent \& Fuzzy Systems, 35(2), 1209-1224. doi:10.3233/JIFS-169666

NovAtel. (2003). GPS Position Accuracy Measures. Retrieved from https://www.novatel.com/assets/Documents/ Bulletins/apn029.pdf

Pandis, N. (2016). Multiple linear regression analysis. American Journal of Orthodontics and Dentofacial Orthopedics, 149(4), 581. doi:10.1016/j.ajodo.2016.01.012 PMID:27021463

Qi, Y., Kobayashi, H., \& Suda, H. (2006). Analysis of wireless geolocation in a non-line-of-sight environment. IEEE Transactions on Wireless Communications, 5(3), 672-681. doi:10.1109/TWC.2006.1611097

Razally, F. \& Davies, P. (2015). Mobile Handset Testing. OFCOM, The UK Communication Regulator. Retrieved from https://www.ofcom.org.uk/_data/assets/pdf_file/0015/72231/mobile_handset_testing_1v01.pdf

Reed, J., Krizman, J., Woerner, B., \& Rappaport, T. (1998). An overview of the challenges and progress in meeting the e-911 requirement for location service. IEEE Personal Communications Magazine, 5(3), 30-37.

Shang, J., Hu, X., Gu, F., Wang, D., \& Yu, S. (2015). Improvement schemes for indoor mobile location estimation: A survey. Mathematical Problems in Engineering, 2015, 1-32. doi:10.1155/2015/397298

Surendra, S., Reenu, M., \& Chun, K. P. (2019). Mobile Location Estimation Based on Received Signal Strength using Circular Approach. Journal of Engineering and Applied Sciences (Asian Research Publishing Network), 14(1), 3922-3926. doi:10.3923/jeasci.2019.3922.3926

Takenga, C.M. \& Kyamakya, K. (2007). Location fingerprinting in GSM network and impact of data preprocessing. 
Tong, K. (2015). Location Estimation in Wireless Communication Systems. University of Western Ontario, Canada.

Venkatraman, S., \& Caffery, J. (2004). Hybrid TO/AOA techniques for Mobile Location in Non-Line-of-Sight Environments. In Proc. IEEE-WCNC (Vol. 1, pp. 274-278). IEEE. doi:10.1109/WCNC.2004.1311556

Walpole, R. E., \& Myers, R. H. (1985). Probability and Statistics for Engineers and Scientists (3rd ed.). New York: Macmillan.

Zhou, J., \& Kee-Yin Ng, J. (2006). Analysis of Statistical Estimation for Mobile Location Estimation. Kowloon Tong, Hong Kong: Department of Computer Science, Baptist University.

Longinus Sunday Ezema graduated with B. Eng in Electrical and Electronic Engineering from Nnamdi Azikiwe University, Awka, Nigeria in 2004, obtained a Master of Science (M.Sc) in Telecommunication System from Blekinge Institute of Technology (BTH), Sweden in 2007 and a Ph.D in Electronic Engineering (Communication Engineering Option) from University of Nigeria in 2018. He is a Lecturer 1 in the Electrical and Electronic Engineering Department, Federal University of Technology, Owerri, Nigeria. He is solution focused, dynamic and creative lecturer, committed with the learning and the students' progress, who searches to motivate human values and to develop basic abilities like research, working in a group and the making of decisions. He has ten (10) years postgraduate experience in operations management, research and lecturing. He also worked as Operation Management Coordinator, Golden Shield Company, London, United Kingdom. He is currently researching on radio location, cloud computing and mobility load balancing for congestion avoidance using artificial intelligent in 3GPP LTE network. He is a Member Nigerian Society of Engineers (NSE), Member National Society of Black Engineers (NSBE) and a COREN Registered Engineer. Recipient of the Federal Government of Nigeria Grant through Nigerian Tertiary Education Trust Fund, PhD research, 2015. 